\title{
A New Adaptive Kalman Estimator for Detection and Isolation of Multiple Faults Integrated in a Fault Tolerant Control
}

\author{
H. Jamouli ${ }^{1}$ and D. Sauter ${ }^{2}$ \\ ${ }^{1}$ Ecole Nationale des Sciences Appliquées Université Ibn Zohr, Agadir, Morocco \\ ${ }^{2}$ Centre de Recherche en Automatique de Nancy, Université Henri Poincaré Nancyl, \\ CNRS UMR 7039, BP 239, 54506 Vandoeuvre Cedex, France
}

Correspondence should be addressed to H. Jamouli, jamouli@ensa-agadir.ac.ma

Received 17 March 2010; Revised 4 July 2010; Accepted 27 October 2010

Academic Editor: Edwin Chong

Copyright (C) 2010 H. Jamouli and D. Sauter. This is an open access article distributed under the Creative Commons Attribution License, which permits unrestricted use, distribution, and reproduction in any medium, provided the original work is properly cited.

For sequential jumps detection, isolation, and estimation in discrete-time stochastic linear systems, Willsky and Jones (1976) have developed the Generalized Likelihood Ratio (GLR) test. After each detection and isolation of one jump, the treatment of another possible jump is obtained by a direct state estimate and covariance incrementation of the Kalman filter originally designed on the jump-free system. This paper proposes to extend this approach from a state estimator designed on a reference model directly sensitive to system changes. We will show that the obtained passive GLR test can be easily integrated in a Fault Tolerant Control System (FTCS) via a control law designed in order to asymptotically reject the effect of sequential jumps.

\section{Introduction}

First derived in a completely recursive form by Willsky and Jones [1], the GLR test has been used in a wide variety of applications including the detection of sensor and actuator failures [1-3], electrocardiogram analysis [4], geophysical signal processing [5], and freeways supervision [6]. For sequential jumps detection in discrete-time stochastic linear systems, the GLR test is made of the following steps:

(1) detection and isolation of one possible jump by applying a GLR detector on the innovation sequence of the Kalman filter designed on the jump-free system,

(2) updating of the Kalman filter using the jump magnitude estimate given by the GLR detector,

(3) go to the step 1 to detect, isolate, and estimate another possible jump from measurements immediately available after the detection time of the last jump.

The first part of this paper revisits the standard GLR test of Willsky and Jones [1] with respect to the fault detectability indexes [7] describing the time delay between the occurrence of a jump and its effect on measurements.

The second part presents a new GLR test based on the augmented state Kalman estimator designed on the least favorable reference model including all the states of hypothetical jumps. This is not a new approach in the area of an FDI in dynamic systems-based observer. The wellknown fault detection filter originally developed by Beard [8] is also designed from a fixed reference model including all the possible faults. The sequential multiple decision theory is not complete and this paper has not the pretention to give a unique solution of this general problem but only a usefull solution focussed on the integration of the obtained FDI scheme in an FTCS.

Over the last two decades, growing demand for reliability, maintainability in dynamic systems has drawn significant research in the area of Fault detection and isolation (FDI) (see $[9,10]$ and references therein). A fault tolerant control system (FTCS) is a control system which possesses the ability to accommodate system component failures automatically. The existing methods for reconfigurable controller design can be classified as linear quadratic regulator $[11,12]$, eigenstructure assignment [13], multiple model [14], adaptive 
control [15], pseudoinverse [16], and model following [17] and recently, [18-20] have proposed a kind of controllers stabilizing for fault tolerant control. For fuzzy system [21] a new approach of FTC is developed taking into account uncertainties of system model. In this context, the last part of the paper proposes to integrate our passive GLR test in an FTCS via a control law of form $u_{k}=u_{k}^{n}-G \hat{v}_{k}$, where the nominal control law $u_{k}^{n}=-\bar{L}_{\bar{x}}$ will be corrected online by the additional input signal $G \hat{\nu}_{k}$ depending on the jump magnitude prediction $\hat{v}_{k}$. This control law will be designed from the two-stage Kalman filter of Friedland [22] allowing the state prediction $\hat{\bar{x}}_{k}$ of the jump-free system to be available at each step of the processing. The additional input signals $G \hat{v}_{k}$ superimposed to the nominal control inputs will be computed so that the influence of the jumps on the regulated variables is asymptotically rejected.

\section{The A Priori Assumptions}

The model of the jump-free system, noted $h_{0}$, is given by

$$
\begin{gathered}
x_{k+1}=A x_{k}+B u_{k}+w_{k}, \\
y_{k}=C x_{k}+v_{k},
\end{gathered}
$$

where $x_{k} \in \mathfrak{R}^{n}$ is the state vector, $y_{k} \in \mathfrak{R}^{m}$ the measurement vector, $u_{k} \in \mathfrak{R}^{r}$ the input vector. The zero mean white Gaussian noises $w_{k}$ and $v_{k}$ satisfy

$$
E\left\{\left[\begin{array}{l}
\omega_{k} \\
v_{k}
\end{array}\right]\left[\begin{array}{ll}
\omega_{j}^{T} & v_{j}^{T}
\end{array}\right]\right\}=\left[\begin{array}{cc}
W & 0 \\
0 & V
\end{array}\right] \delta_{k, j},
$$

where $W \geq 0$ and $V>0$ and the Gaussian initial state $x_{0}, \hat{\bar{x}}_{0}=E\left(x_{0}\right)$ and $\bar{P}_{0}=E\left\{\left(x_{0}-E\left(x_{0}\right)\right)\left(x_{0}-E\left(x_{0}\right)\right)^{T}\right\}$, $\delta_{k, j}$ is the Kronecker operator. The jump hypotheses $h_{i}$ for $i \in[1, \ldots, N]$ are modelized by

$$
\begin{gathered}
x_{k+1}=A x_{k}+B u_{k}+f_{i}(k, r) v(k, r)+w_{k}, \\
y_{k}=C x_{k}+v_{k},
\end{gathered}
$$

where $f_{i}(k, r)$ is the fault distribution vector, $\nu(k, r)$ the scalar fault magnitude, and $r$ the unknown time of failure occurrence. Without lost of generality, the fault magnitude $\nu(k, r)$ is assumed to follow a constant bias model $v(k, r)=v,(k \geq$ $r)$ with step profile: $f_{i}(k, r)=f_{i},(k \geq r)$ and $f_{i}(k, r)=0$, $(k<r)$. Based on the results of Tanaka [23] and Caglyan [24] (not developed in this paper), the detectability and distinguishability conditions of multiple jumps are assumed to be satisfied $\operatorname{rank}\left[C A^{\mu_{1}-1} f_{1} \cdots C A^{\mu_{i}-1} f_{i} \cdots C A^{\mu_{N}-1} f_{N}\right]=N$

$$
\operatorname{rank}\left[\begin{array}{cccccc}
I-A & f_{1} & \cdots & f_{i} & \cdots & f_{N} \\
C & & & 0 & &
\end{array}\right]=n+N .
$$

Let $\mu_{i}=\min \left(s: C A^{s-1} f_{i} \neq 0, s=1,2, \ldots\right)$ for $i \in[1, \ldots, N]$.

The jump detectability indexes [7], and assume that $\rho_{i}<\infty$ signifying that the first information about the jump $h_{i}$, appearing at time $r$, will be present on measurement $y_{r+\rho_{i}}$.
In the case where the jumps may occur relatively infrequently, the most likelihood reference model at the beginning of the processing is the model of $h_{0}$. So, under the stability and convergence conditions

$$
\begin{aligned}
& \operatorname{rang}\left[\begin{array}{c}
z I-A \\
C
\end{array}\right]=n, \quad|z| \geq 1, \\
& \operatorname{rang}\left[-e^{j w} I+A W^{1 / 2}\right]=n, \quad w \in[0,2 \pi] \text {. }
\end{aligned}
$$

The jumps may occur relatively infrequently, and we assume that the nominal control law $u_{k}^{n}=-\bar{L} \hat{\bar{x}}_{k}$ satisfying the nominal system performances has been designed on the jump-free system (1) via for example LQG approach on infinite horizon [25]. No a priori information is available concerning the jump's scenario: after the detection of one or several jumps, no information is available to focus our attention on another particular jump. The only assumption is that two jumps cannot appear simultaneously but only sequentially with a sufficient time delay to allow their realtime recursive treatment on a sliding window $W=[k-M \leq$ $r \leq k]$ of size $M$. In other words window $W$ may include occurrence time $r$ of one jump only. So, $M$ will be chosen in accordance with the maximum available computational time and with the minimum time delay between the occurrence of two jumps depending to the practical application of our works.

\section{The $n$-Order Adaptive Kalman Estimator of Willsky and Jones}

This chapter revisits the GLR test developed by Willsky and Jones [1] in relation with multihypotheses GLR test described in Willsky [26]. The a priori reference model at the beginning of the processing is the model $h_{0}$. Under the stability and convergence conditions (6), (7), the Kalman filter which gives the maximum likelihood state prediction of the system is given by

$$
\begin{gathered}
\hat{\bar{x}}_{k+1}=A \hat{\bar{x}}_{k}+B u_{k}+\bar{K}_{k}\left(y_{k}-C \bar{x}_{k}\right), \\
\bar{P}_{k+1}=\left(A-K_{k} C\right) \bar{P}_{k}\left(A-K_{k} C\right)^{T}+W+\bar{K}_{k} V \bar{K}_{k}^{T}, \\
\bar{H}_{k}=C \bar{P}_{k} C^{T}+V \\
\bar{K}_{k}=A \bar{P}_{k} C^{T} \bar{H}_{k}^{-1} .
\end{gathered}
$$

The additive effect of jump $h_{i}$ on the state prediction error $e_{k+1}=x_{k+1}-\hat{\bar{x}}_{k+1}$ and on the innovation sequence $\bar{\gamma}=y_{k}-$ $C \hat{\bar{x}}_{k}$ can be expressed as

$$
\begin{aligned}
e_{k+1} & =\tilde{e}_{k+1}+\zeta_{i}(k+1, r), \\
\bar{\gamma}_{k} & =\tilde{\gamma}_{k+1}+\varrho_{i}(k, r) \nu
\end{aligned}
$$

where $\tilde{e}_{k+1}$ and $\tilde{\gamma}_{k}$ represent the state prediction error and the innovation sequence on the jump-free system and where 
$\zeta_{i}(k, r)$ and $\varrho_{i}(k, r)$ describe the additive effect of the jump satisfying

$$
\begin{gathered}
\zeta_{i}(k+1, r)=\left(A-\bar{K}_{k} C\right) \zeta_{i}(k, r)+f_{i}, \quad \zeta_{i}(r, r)=0, \\
\varrho_{i}(k, r)=C \zeta_{i}(k, r) .
\end{gathered}
$$

In Willsky [26] or Basseville and Nikiforov [27], $\zeta_{i}(k, r)=\alpha_{i}(k, r)-\beta_{i}(k, r)$, where $\alpha_{i}(k, r)$ and $\beta_{i}(k, r)$ describe the additive effect of the jump on the state vector of the system and on the state vector of the Kalman filter, respectively. Our formulation shows that the jump signatures (14) only depend to the state prediction error of the Kalman filter and thus are decoupled from $u_{k}$ (this property will be used in the design of the FTCS). The jump hypothesis $h_{i}$ can be confronted to the "no-jump" hypothesis $h_{0}$ as

$$
\begin{gathered}
H_{0}: E\left(\bar{\gamma}_{t}\right)=0, \quad t<r, \\
H_{i}: E\left(\bar{\gamma}_{t}\right)=\varrho_{i}(t, r) v, \quad k \geq t \geq r, \quad i \in[1, \ldots, N] .
\end{gathered}
$$

Since $E\left\{\left(\bar{\gamma}_{k-t}-E\left(\bar{\gamma}_{k-t}\right)\right)\left(\bar{\gamma}_{k}-E\left(\bar{\gamma}_{k}\right)\right)^{T}\right\}=0$ for all $t<k$, the likelihood ratio between (15) and (16) gives

$$
\lambda_{i}(k, r, v)=\frac{P\left(\bar{\gamma}_{0} / h_{0}\right) \cdots P\left(\bar{\gamma}_{r_{i}+\rho_{i}} / h_{i}\right) \cdots P\left(\bar{\gamma}_{k} / h_{i}\right)}{P\left(\bar{\gamma}_{0} / h_{0}\right) \cdots P\left(\bar{\gamma}_{r_{i}+\rho_{i}} / h_{0}\right) \cdots P\left(\bar{\gamma}_{k} / h_{0}\right)} .
$$

From (14), we have $\varrho_{i}(r, r)=\varrho_{i}(r+1, r)=\cdots=\varrho_{i}\left(r+\mu_{i}-\right.$ $1, r)=0$ leading to

$$
\begin{aligned}
& P\left(\frac{\bar{\gamma}_{r_{i}}}{h_{i}}\right)=P\left(\frac{\bar{\gamma}_{r_{i}}}{h_{0}}\right), \\
& P\left(\frac{\bar{\gamma}_{r_{i}+1}}{h_{i}}\right)=P\left(\frac{\bar{\gamma}_{r_{i}+1}}{h_{0}}\right), \ldots, \\
& P\left(\frac{\bar{\gamma}_{r_{i}+\mu_{i}-1}}{h_{i}}\right)=P\left(\frac{\bar{\gamma}_{r_{i}}+\mu_{i}-1}{h_{0}}\right),
\end{aligned}
$$

and the likelihood ratio (17) can be rewritten as

$$
\begin{aligned}
\lambda_{i}(k, r, v) & =\frac{P\left(\bar{\gamma}_{r_{i}+\mu_{i}} / h_{i}\right) \cdots P\left(\bar{\gamma}_{k} / h_{i}\right)}{P\left(\bar{\gamma}_{r_{i}+\mu_{i}} / h_{0}\right) \cdots P\left(\bar{\gamma}_{k} / h_{0}\right)} \\
& =\frac{\exp \left((-1 / 2) \sum_{t=r+\mu_{i}}^{k}\left\|\bar{\gamma}_{t}-\varrho_{i}(t, r) \nu\right\|_{\bar{H}_{t}^{-1}}^{2}\right)}{\exp \left((-1 / 2) \sum_{t=r+\mu_{i}}^{k}\left\|\bar{\gamma}_{t}\right\|_{\bar{H}_{t}^{-1}}^{2}\right)} .
\end{aligned}
$$

Based on $\bar{\gamma}_{0}, \ldots, \bar{\gamma}_{k}$, the maximum likelihood prediction of $v$ conditioned on a particular assumed value of $r$ is given by

$$
\hat{\nu}(k+1, r)=\left[\sum_{t \in r+\mu_{i}}^{k} \varrho_{i}^{T}(t, r) \bar{H}_{t}^{-1} \varrho_{i}(t, r)\right]^{-1} \sum_{t=r+\mu_{i}}^{k} \varrho_{i}^{T}(t, r) \bar{H}_{t}^{-1} \bar{\gamma}_{t},
$$

and the log-likelihood ratio $T_{i}(k, r)=2 \log \left(\lambda_{i}(k, r, \hat{\nu}(k+\right.$ $1, r)))$ can be written as

$$
\begin{gathered}
T_{i}(k, r)=b_{i}(k, r)^{2} a_{i}(k, r)^{-1}, \\
a_{i}(k, r)=\sum_{t=r+\mu_{i}}^{k} \varrho_{i}^{T}(t, r) \bar{H}_{t}^{-1} \varrho_{i}(t, r), \\
b_{i}(k, r)=\sum_{t=r+\mu_{i}}^{k} \varrho_{i}^{T}(t, r) \bar{H}_{t}^{-1} \bar{\gamma}_{t} .
\end{gathered}
$$

So, the decision rule of the GLR detector is as follows:

$$
\max _{i \in[1, \ldots, N], \tilde{r} \in[0, \ldots, k]}\left\{T_{i}\left(k, \tilde{r}-\mu_{i}\right)\right\}>\varepsilon,
$$

where $\varepsilon$ is the decision threshold. For a practical implementation of the GLR detector (22), the translated jump occurrence time $\tilde{r}$ must be constrained to belong to the sliding windows $W=[k-M \leq \tilde{r} \leq k]$ of size $M$. In this case, (22) can be rewritten

$$
\max _{i \in[1, \ldots, N], \tilde{r} \in W}\left\{T_{i}\left(k, \tilde{r}-\mu_{i}\right)\right\}>\varepsilon .
$$

If $\max \left\{T_{i}\left(k, \tilde{r}-\mu_{i}\right\}>\mathcal{E}\right.$, then one jump is detected at time $k$, isolated from $(j, \hat{\widetilde{r}})=\arg \max \left\{T_{i}\left(k, \tilde{r}-\mu_{i}\right)\right\}$, where $\hat{r}=\hat{\widetilde{r}}-\mu_{j}$ is the estimation of the jump occurrence time and where

$$
\begin{gathered}
\hat{\nu}(k+1, \hat{r})=a_{j}(k, \hat{r})^{-1} b_{j}(k, \hat{r}), \\
P^{v}(k+1, \hat{r})=a_{j}(k, \hat{r})^{-1}
\end{gathered}
$$

represent the maximum likelihood prediction of the jump magnitude based on measurements until time $k$ under the assumption that $v$ has an infinite a priori covariance.

The second step of the standard GLR test, intuitively obtained from relation (12), consists in updating the Kalman filter ( 8 ) by prediction and covariance incrementation of the Kalman filter (8) as follows:

$$
\begin{gathered}
\hat{x}_{k+1}^{\text {new }}=\bar{x}_{k+1}^{\text {old }}+\zeta_{j}(k+1, \hat{r}) \hat{\nu}(k+1, \hat{r}), \\
P_{k+1}^{\text {new }}=\bar{P}_{k+1}^{\text {old }}+\zeta_{j}(k+1, \hat{r}) P \zeta_{j}(k+1, \hat{r})^{T},
\end{gathered}
$$

where the new state prediction $\left(\hat{x}_{k+1}^{\text {new }}, P_{k+1}^{\text {new }}\right)$ is substituted in the Kalman filter (13) (see Figure 1 in Willsky and Jones [1]). In the original version of the GLR test, the state variables of the matched filters $\zeta_{i}(k, r)$ for $i \in[1, \ldots, N]$ are reinitialized to zero immediately following the filter incrementation leading to consider $\left(\hat{x}_{k+1}^{\text {new }}, P_{k+1}^{\text {new }}\right)$ as a new initialization of the Kalman filter more appropriate than the initial value given at the beginning of the processing [27]. With this implementation, (24) cannot be used to improve the jump estimation from measurements available after its detection leading to choose the threshold level $\varepsilon$ by reaching a compromise between fast detection and accurate estimation.

To avoid this tradeoff, we can modify this implementation in such a way that $\left(\hat{x}_{k+1}^{\text {new }}, P_{k+1}^{\text {new }}\right)$ is not substituted in the Kalman filter but used to generate the auxiliary innovation 
sequence $\gamma_{k}=y_{k}-C \hat{x}_{k}^{\text {new }}$ and its covariance matrix $H_{k}=$ $C P_{k}^{\text {new }} C^{T}+V$ expressed

$$
\begin{gathered}
\gamma_{k}=\bar{\gamma}_{k}-\varrho_{j}(k, \hat{r}) \hat{\nu}(k, \hat{r}), \\
H_{k}=\bar{H}_{k}+\varrho_{j}(k, \hat{r}) P^{v}(k, \hat{r}) \varrho_{j}(k, \hat{r})^{T},
\end{gathered}
$$

where $\bar{\gamma}_{k}$ and $\bar{H}_{k}$ represent the innovation sequence of the Kalman filter (8) and its covariance matrix. Now decoupled to the updating strategy, the Kalman filter, ((8), (24), and (14)) can be used to improve the jump estimation from measurements available after its detection time, and another possible jump can then be treated by the following GLR detector:

$$
\max _{i \in[1, \ldots, N], i \neq j r \in W}\left\{\widetilde{T}_{i}\left(k-\rho_{i}, \widetilde{r}\right)\right\}>\varepsilon
$$

with

$$
\begin{gathered}
\tilde{T}_{i}(k, r)=\tilde{b}_{i}(k, r)^{2} \tilde{a}_{i}(k, r)^{-1}, \\
\tilde{a}_{i}(k, r)=\sum_{t=r+\rho_{i}}^{k} \varrho_{i}^{T}(t, r) H_{t}^{-1} \varrho_{i}(t, r), \\
\tilde{b}_{i}(k, r)=\sum_{t=r+\rho_{i}}^{k} \varrho_{i}^{T}(t, r) H_{t}^{-1} \gamma_{t},
\end{gathered}
$$

where the jump signatures $\varrho_{i}(t, r)$ are computed from (14) after having reinitialized $\zeta_{i}(k, r)$ for $i \neq j$ to zero immediately after the detection time of the first jump.

\section{The $N+n$ Order Adaptive Kalman Estimator}

Our passive GLR test will not be designed from the jumpfree system but on a reference model including all the hypothetical states of jumps that we wish to detect and isolate. This model will not be considered as the true model of the faulty system since the jump hypotheses $((4),(5))$ are always assumed to occur relatively infrequently. Let

$$
\begin{gathered}
X_{k+1}=\bar{A} X_{k}+\bar{B} u_{k}+\bar{\Gamma} w_{k}, \\
y_{k}=\bar{C} X_{k}+v_{k}
\end{gathered}
$$

with $\bar{A}=\left[\begin{array}{ll}A & F \\ 0 & 1\end{array}\right], \bar{B}=\left[\begin{array}{l}B \\ 0\end{array}\right], \bar{\Gamma}=\left[\begin{array}{l}I \\ 0\end{array}\right], \bar{C}=\left[\begin{array}{ll}C & 0\end{array}\right], X_{k}=\left[\begin{array}{l}x_{k} \\ v_{k}\end{array}\right]$ the augmented state model including all jump's states $v_{k}=$ $\left[v_{k}^{1} \cdots v_{k}^{j} \cdots v_{k}^{N}\right]$ that we wish to detect and isolate. From (30), the jump hypothesis $h_{j}$ described by (2) can be viewed as an impulsive abrupt change on the $j^{\text {th }}$ hypothetical jump's state, modelized as

$$
v_{k+1}^{j}=v_{k}^{j}+\Delta \nu \delta_{k r}, \quad \forall j \in[1, \ldots, N],
$$

where $r$ is the unknown occurrence time of impulsive abrupt change, $\Delta v$ the jump's state increment, and $\delta_{k \mathrm{r}}$ is the Kronecker operator.

Substituting (32) in (30), we obtain the impulsive jump hypotheses, noted $h_{j}^{\Delta}$, described by

$$
\begin{gathered}
X_{k+1}=\bar{A} X_{k}+\bar{B} u_{k}+f_{j}^{\Delta}(k, r) \Delta \nu(k, r)+\bar{\Gamma} w_{k}, \\
y_{k}=\bar{C} X_{k}+v_{k}
\end{gathered}
$$

with $\Delta v(k, r)=\Delta \nu \delta_{k r_{i}}, f_{j}^{\Delta}(k, r)=f_{j}^{\Delta} \Delta v\left(k, r_{i}\right)$ and $\bar{\Gamma}=$ $\left[\begin{array}{ll}0 & I_{j}^{T}\end{array}\right]^{T}$ where $I_{j}^{T}=\left[\begin{array}{lllll}0 & \cdots & 1 & \cdots & 0\end{array}\right]$ has one at the $j$ th position and zero elsewhere. Based on an approach very similar to the modified GLR test of part 3, let

$$
\begin{gathered}
\hat{X}_{k+1}=\bar{A} \hat{X}_{k}+\bar{B} u_{k}+K_{k}\left(y_{k}-\bar{C} \hat{X}_{k}\right) \\
\Omega_{k+1}=\bar{A} \Omega_{k} \bar{A}^{T}+\bar{\Gamma} W \bar{\Gamma}^{T}-\bar{A}_{k} \Omega_{k} \bar{C}^{T}\left(\bar{C} \Omega_{k} \bar{C}^{T}+V\right)^{-1} \bar{C} \Omega_{k} \bar{A}^{T} \\
K_{k}=\bar{A} \Omega_{k} \bar{C}^{T} H_{k}^{-1} \\
H_{k}=\bar{C} \Omega_{k} \bar{C}^{T}+V
\end{gathered}
$$

the augmented state Kalman filter designed on the reference model (30), (31). The additive effect of the impulsive jump hypothese $h_{i}^{\Delta}$ on the state prediction error and on the innovation sequence of the augmented state Kalman filter propagates as

$$
\begin{gathered}
e_{k+1}=\tilde{e}_{k+1}+\zeta_{j}^{\Delta}(k+1, r) \Delta \nu, \\
\gamma_{k}=\tilde{\gamma}_{k+1}+\varrho_{j}^{\Delta}(k, r) \Delta \nu,
\end{gathered}
$$

where $\tilde{e}_{k+1}$ and $\tilde{\gamma}_{k}$ represent the state prediction error and the innovation sequence on the jump-free system and where $\zeta_{j}^{\Delta}(k+1, r)$ and $\varrho_{j}^{\Delta}(k, r)$ propagate as

$$
\begin{aligned}
\zeta_{j}^{\Delta}(k+1, r)= & \left(\bar{A}-\bar{K}_{k} \bar{C}\right) \zeta_{j}^{\Delta}(k, r), \zeta_{j}^{\Delta}(r, r)=f_{j}^{\Delta}, \\
& \varrho_{j}^{\Delta}(k, r)=\bar{C} \zeta_{j}^{\Delta}(k, r) .
\end{aligned}
$$

Based on the part 3, the following GLR detector is then derived

$$
\max _{j \in[1, \ldots, N], \tilde{r} \in W}\left\{T_{j}^{\Delta}\left(k, \tilde{r}-\mu_{j}\right)\right\}>\varepsilon
$$

with

$$
\begin{gathered}
T_{j}^{\Delta}(k, r)=b_{j}^{\Delta}(k, r)^{2} a_{j}^{\Delta}(k, r)^{-1}, \\
a_{j}^{\Delta}(k, r)=\sum_{t=r+\mu_{j}}^{k} \varrho_{j}^{\Delta T}(t, r) H_{t}^{-1} \varrho_{j}^{\Delta}(t, r), \\
b_{j}^{\Delta}(k, r)=\sum_{t=r+\mu_{j}}^{k} \varrho_{j}^{\Delta T}(t, r) H_{t}^{-1} \gamma_{t} .
\end{gathered}
$$

If $\max _{j, \tilde{r}}\left\{T_{j}^{\Delta}\left(k, \tilde{r}-\mu_{j}\right\}>\varepsilon\right.$ then $(i, \hat{\widetilde{r}})=\arg \max _{j, \tilde{r}}\left\{T_{j}^{\Delta}(k, \tilde{r}-\right.$ $\left.\left.\mu_{j}\right)\right\}$, and the impulsive jump $h_{i}^{\Delta}$ is declared to be occurred at time, where $\hat{r}=\hat{\widetilde{r}}-\mu_{i}$ where

$$
\begin{gathered}
\Delta \hat{\nu}(k+1, \hat{r})=a_{i}^{\Delta}(k, \hat{r})^{-1} b_{i}^{\Delta}(k, \hat{r}), \\
P^{\Delta \nu}(k+1, \hat{r})=a_{i}^{\Delta}(k, \hat{r})^{-1}
\end{gathered}
$$

represent the maximum likelihood prediction of the jump increment $\Delta \nu$ (under the assumption that $\Delta \nu$ has an infinite 
a priori covariance). At the detection time of the first jump, the tracking ability of the augmented state Kalman filter (34) can be improved from the updating strategy of Willsky and Jones [1] as

$$
\begin{gathered}
\hat{X}_{k+1}^{\text {new }}=\hat{X}_{k+1}^{\text {old }}+\zeta_{i}^{\Delta}(k+1, \hat{r}) \Delta \hat{\nu}(k+1, \hat{r}), \\
\Omega_{k+1}^{\text {new }}=\Omega_{k+1}^{\text {old }}+\zeta_{i}^{\Delta}(k+1, \hat{r}) P^{\Delta \nu}(k+1, \hat{r}) \zeta_{i}^{\Delta}(k+1, \hat{r})^{T} .
\end{gathered}
$$

In our case, the state of the matched filters given by $\zeta_{j}^{\Delta}(k, r)$ is spanned in the trajectory space of the augmented state Kalman filter's prediction errors. So, (45) and (46) substituted in the augmented state Kalman filter (34) improve its tracking ability without producing a possible instability on the resulting filter (under the stability and convergence conditions of the augmented state Kalman filter given by (6) and (7)). The treatment of another impulsive jump is then obtained by applying GLR detector (41) on the resulting filter after having reinitialized $\zeta_{j}^{\Delta}(k, r)=0$, for all $j \in$ $[1, \ldots, N]$ immediately after the filter incrementation. The new initialization (14) allows $E\left(\hat{X}_{k}^{\text {new }}\right)$ to reach the true state of the system very quickly (and $E\left(\gamma_{t}\right)$ to reach zero for jump compensation, consequently) avoiding the detection of the same jump several times. From an inductive reasoning, the passive GLR test is then derived and consists of the following steps:

(1) detection, isolation, and estimation of one impulsive jump with the GLR detector (41),

(2) updating of the augmented state Kalman filter (34) with (45) to improve its tracking,

(3) going to the step 1.

The sequential multiple decision theory is not complete, and the choice of the threshold level $\varepsilon$ is not studied in this paper. However, some simulation results not presented in this paper show that only statistical tuning parameter $\varepsilon$ can be fixed at the beginning of the processing (this is not the threshold level which is adaptive but the augmented Kalman filter).

To show that the passive GLR test is less powerful than the GLR test of Willsky and Jones [1] (for a same fixed decision threshold) a very simple proof can be given on the treatment of the first jump. The reader can easily show that

$$
\delta_{i}(k, r) \geq \delta_{j}^{\Delta}(k, r)
$$

where $\delta_{i}(k, r)=\left[\sum_{t=r+\mu_{i}}^{k} \varrho_{j}^{T}(t, r) \bar{H}_{t}^{-1} \varrho_{j}(t, r)\right] \nu^{2}$ is the signal to noise ratio of the Willsky and Jones GLR test and where $\delta_{j}^{\Delta}(k, r)=\left[\sum_{t=r+\mu_{j}}^{k} \varrho_{j}^{\Delta T}(t, r) \bar{H}_{t}^{-1} \varrho_{j}^{\Delta}(t, r)\right] v^{2}$ is derived from (41) with $\Delta v=\nu$.

However, our passive GLR test is more robust with respect to the nondetections and false alarms rates: on the least favorable model (30), the good postmodel of the faulty system has no any sense. Do not confuse the adaptation of the filter on its least favorable model (passive GLR test) and the adaptation of the most favorable model on the true system (not studied here).

\section{Results}

To illustrate the proposed approach we considered the following system described by the matrix

$$
\begin{gathered}
A=\left[\begin{array}{cccc}
0.6 & 0.2 & 0 & 0 \\
0 & 0.2 & 0.1 & 0 \\
0 & 0 & 0.4 & 0.1 \\
0 & 0 & 0 & 0.5
\end{array}\right], \quad B=\left[\begin{array}{ll}
0 & 1 \\
1 & 0 \\
0 & 1 \\
1 & 0
\end{array}\right], \\
C=\left[\begin{array}{llll}
1 & 0 & 0 & 0 \\
0 & 1 & 0 & 0 \\
0 & 0 & 1 & 1
\end{array}\right], \quad F=\left[\begin{array}{ll}
1 & 0 \\
1 & 1 \\
1 & 1 \\
0 & 1
\end{array}\right], \\
W=0.01 * \operatorname{Id}(4,4), \quad V=0.5 * \operatorname{Id}(3,3) .
\end{gathered}
$$

The faults isolability is satisfied with $\operatorname{rank}\left[C A f_{1} C A f_{2}\right]$ and $F=\left[\begin{array}{ll}f_{1} & f_{2}\end{array}\right]$.

The statistical variables describing the performances of the reconfigurable FTCS coincide with the statistical variables describing the performances of the statistical test. So, to simplify the Monte Carlo simulation, the proposed example will be realized in open loop.

In the field of dynamic systems, the signal-to-noise ratio $\delta_{i}(k, r)$ is generally more greater than the signal to noise ratio treated in the fields of electrocardiogram analysis or geophysical signal processing, and the size $M$ of the sliding window $W=[k-M \leq \tilde{r} \leq k]$ can be generally chosen small. In our example, we take $M=0$ (we donnot optimize $\tilde{r}$ at all, and we fix the following rate of false alarms: $P^{F}=0.005$ from a table of Chi-Squared distribution).

First, we suppose one that fault occurred at 350 with magnitude 2; we obtain the following results (Figures 1-20).

In the first case, the results show that the proposed state estimation given by our filter (Figures 6-9) is more adaptive to the fault occurrence than the Willsky algorithm (Figures $1-4)$.

In second case, we suppose two sequential faults occurred at 350 and 400 with magnitudes 2; we obtain the following results (Figures 11-20).

In case of two sequential faults, the GLR detector applied on the passive augmented model allows to detect the first faults at $350 \mathrm{~s}$ and the second at $400 \mathrm{~s}$ (see Figure 20). The Willsky GLR detect just the first fault at 350 and cannot detect the second (see Figure 15).

5.1. Remarks and Discussion. We have also computed the rate of false alarms and the rate of good detections with $10^{5}$ Monte Carlo trials. We have obtained $\hat{P}^{F} \simeq 0,01, \hat{P}_{D} \simeq 0,85$ for the modified GLR test and $\hat{P}^{F} \simeq 0,0055, \hat{P}^{D} \simeq 0,91$ for the passive GLR test clearly much power. We conclude that the passive GLR test is very power when quick detections lead to bad jump estimations and thus very usefull for FTCS to maximize the rate of good decisions specially in regard to the occurrence of a big jump which may greatly affect nominal performance of the system. 


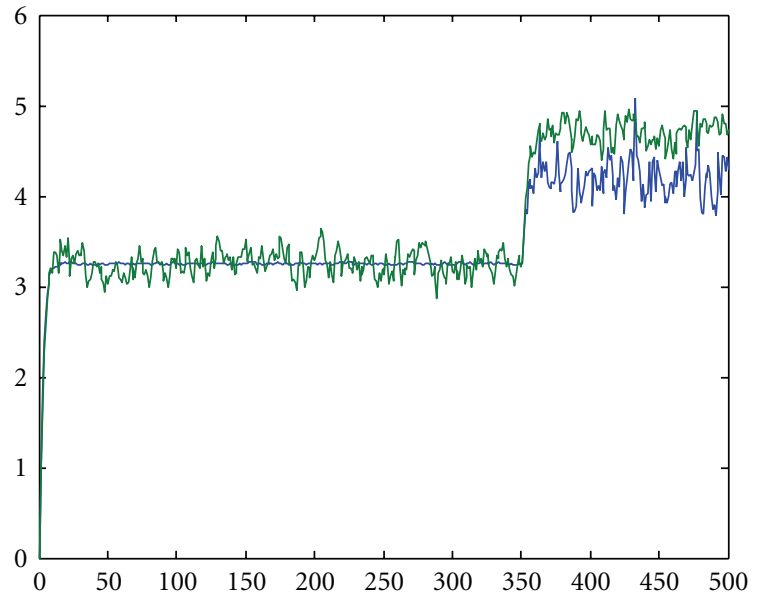

FIGURE 1: First-state component and its estimation given by Willsky algorithm.

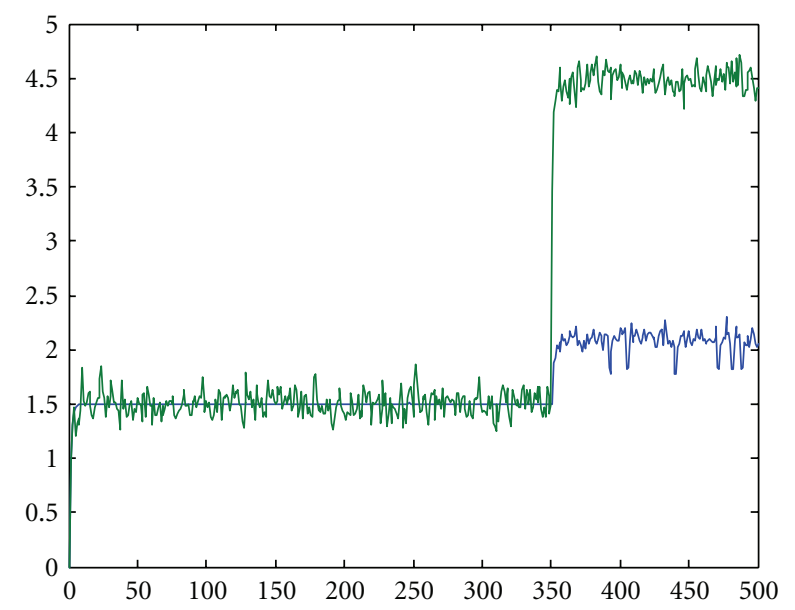

Figure 2: Second-state component and its estimation given by Willsky algorithm.

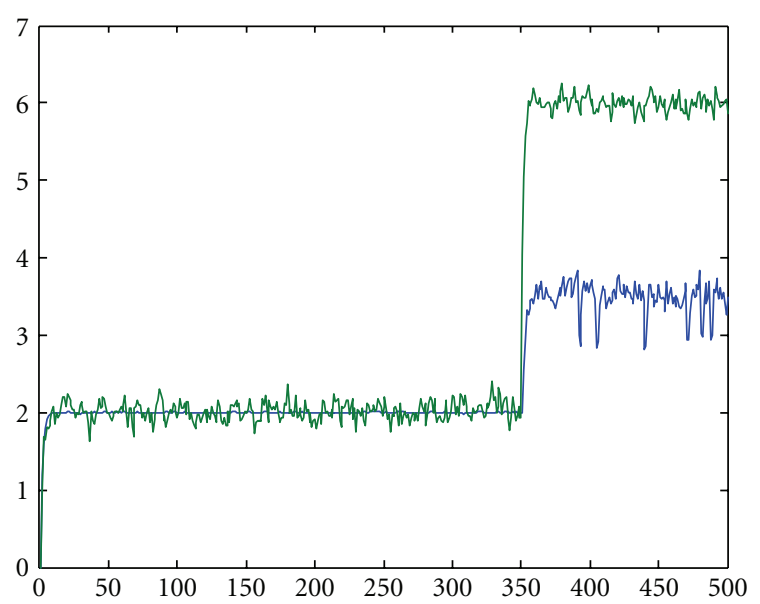

FIgURE 3: Third-state component and its estimation given by Willsky algorithm.

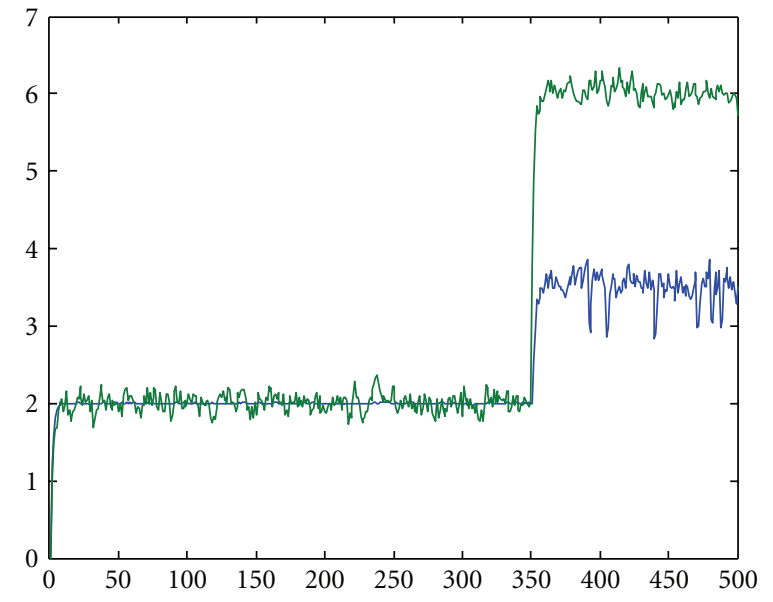

FIGURE 4: Fourth-state component and its estimation given by Willsky algorithm.

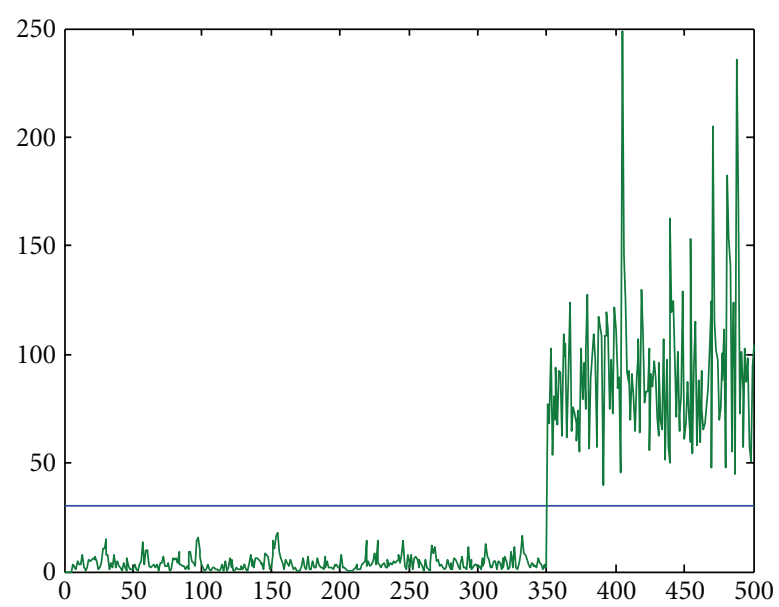

FIGURE 5: GLR test applied on willsky algorithm.

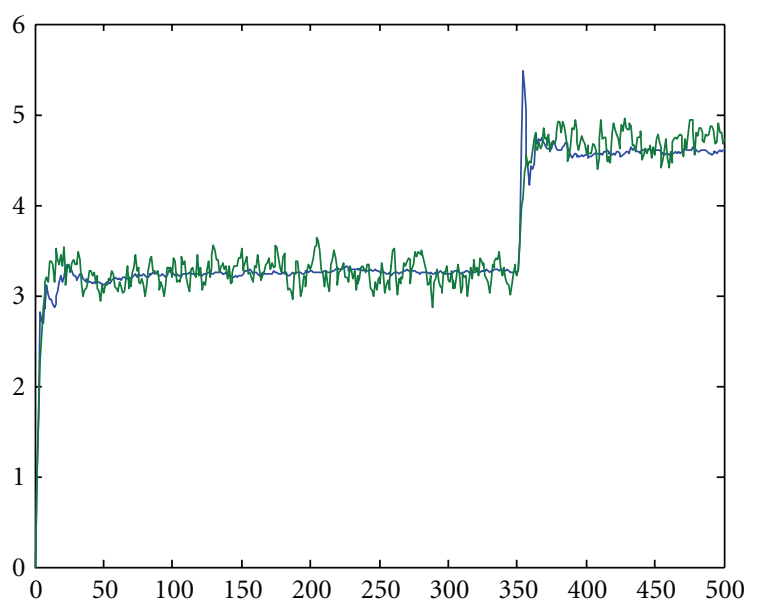

FIGURE 6: First-state component and its estimation given by our adaptive filter algorithm. 




FIGURE 7: Second-state component and its estimation given by our adaptive filter algorithm.

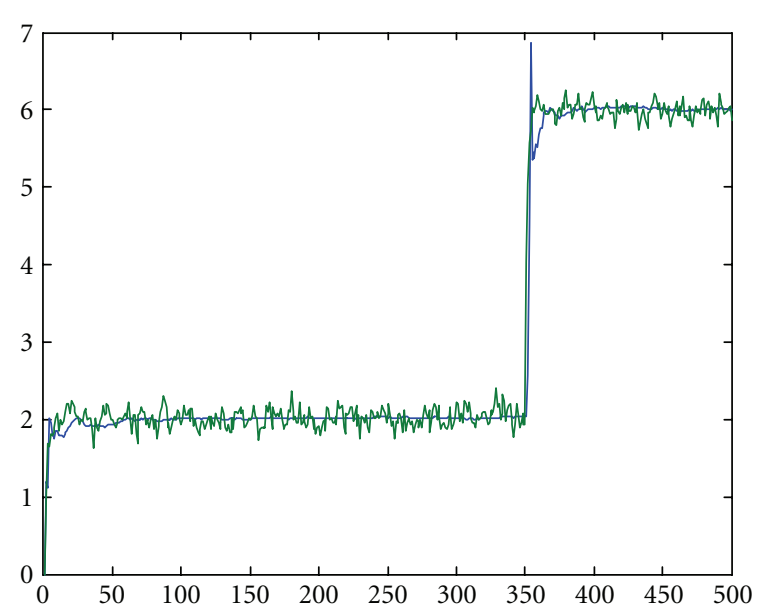

FIgURE 8: Third-state component and its estimation given by our adaptive filter algorithm.



FIGURE 9: Fourth-state component and its estimation given by our adaptive filter algorithm.

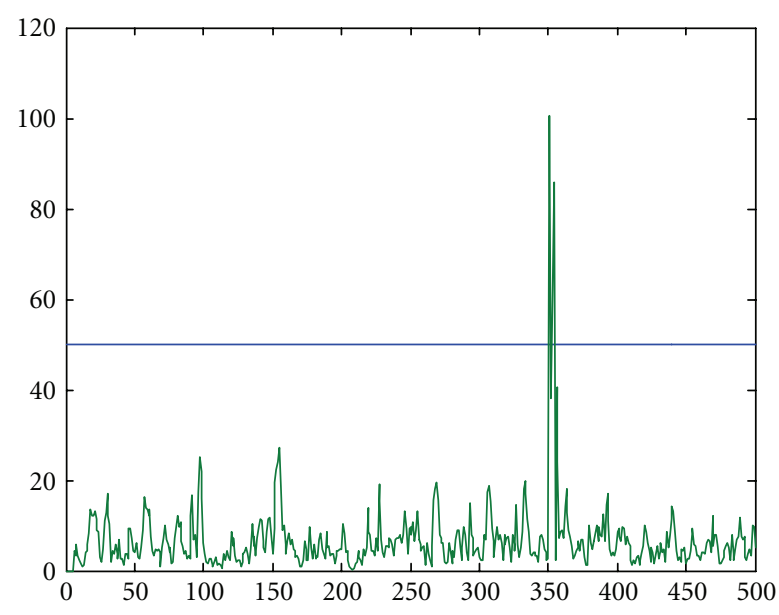

FIGURE 10: GLR test applied to our adaptive filter.

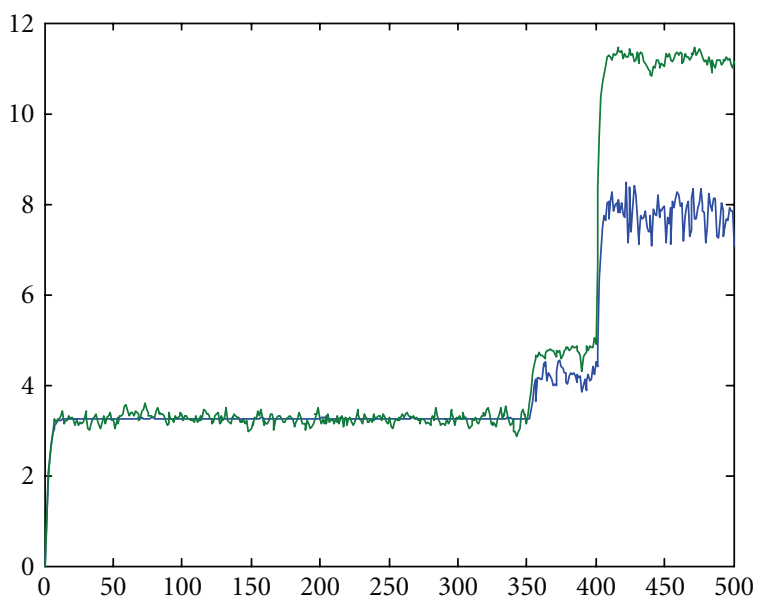

FIGURE 11: First-state component and its estimation given by Willsky algorithm with presence of two sequential faults.



FIGURE 12: Second-state component and its estimation given by Willsky algorithm with presence of two sequential faults. 


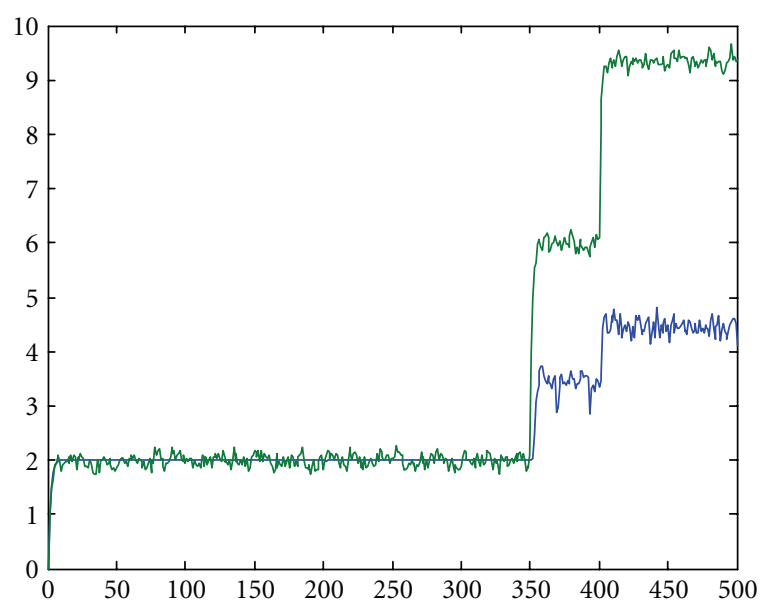

FIgURE 13: Third-state component and its estimation given by Willsky algorithm with presence of two sequential faults.

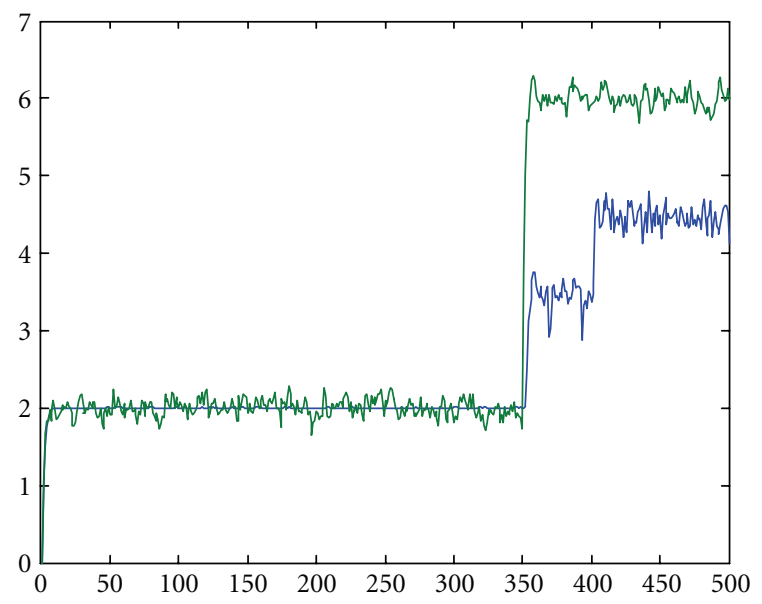

FIGURE 14: Fourth-state component and its estimation given by Willsky algorithm with presence of two sequential faults.

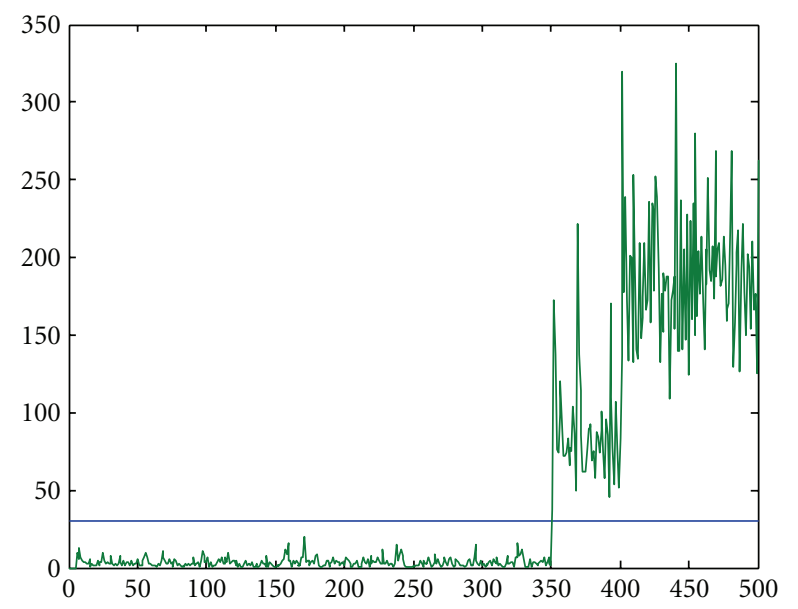

FIGURE 15: GLR test applied to willsky algorithm with presence of two sequential faults.

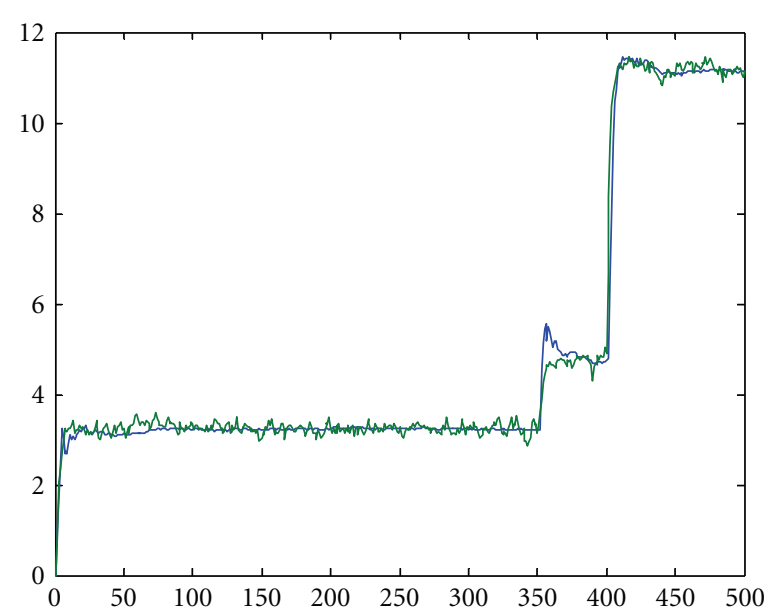

FIGURE 16: First-state component and its estimation given by our adaptive algorithm with presence of two sequential faults.

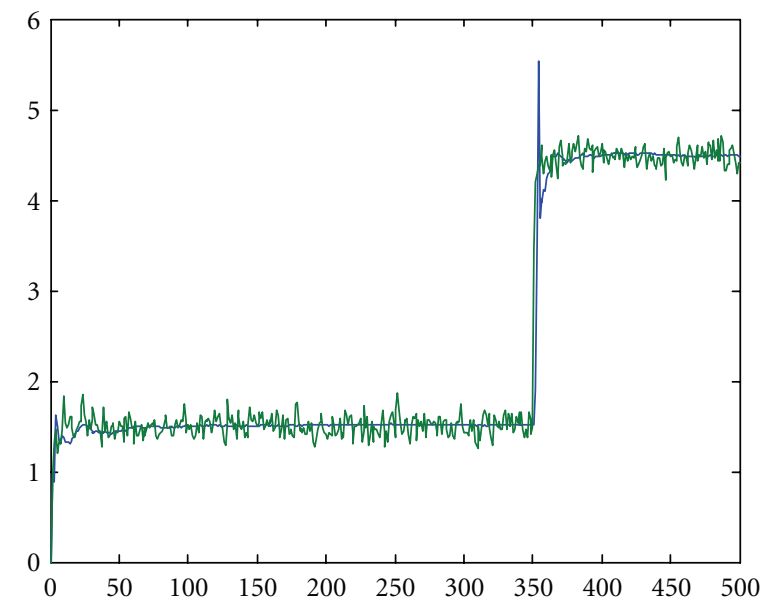

FIGURE 17: Second-state component and its estimation given by our adaptive algorithm with presence of two sequential faults.



FIGURE 18: Third-state component and its estimation given by our adaptive algorithm with presence of two sequential faults. 




FIGURE 19: Fourth-state component and its estimation given by our adaptive algorithm with presence of two sequential faults.

We can improve this approach of detection and isolation with an active GLR based on free model which it will be augmented after each detection and isolation. The faults already detected and isolated will be considered as perturbation, and the we will update the new GLR in order to detect another fault.

The sequential multiple decision theory is not complete, and the choice of the threshold level $\varepsilon$ is not studied in this paper. However, some simulation results not presented in this paper show that only statistical tuning parameter $\varepsilon$ can be fixed at the beginning of the processing (this is not the threshold level which is adaptive but the augmented Kalman filter).

\section{A Reconfigurable Fault Tolerant Control System}

The purpose of the chapter is to show how the passive GLR test can be used in an FTCS. Our FTCS is only designed to reach the unique goal

$$
\underset{k \rightarrow \infty}{E\left(y_{k}\right)=0} \text { under } r<\infty,
$$

in order words to asymptotically reject the effect of jumps on the output of the system (the reference input will be maintained equal to zero avoiding the need of a reconfigurable feedforward control law). The proposed FTCS is based on the passive GLR test integrated via a reconfigurable control law designed on the model

$$
\begin{gathered}
X_{k+1}=\bar{A} X_{k}+\bar{B} u_{k}+\bar{\Gamma} w_{k}, \\
y_{k}=\bar{C} X_{k}+v_{k},
\end{gathered}
$$

where the main problem to reach our goal is that the pair $(\bar{A}, \bar{B})$ has $N$ uncontrollable modes (under $(A, B)$ controllable). The reconfigurable control law of the form $u_{k}=u_{k}^{n}-G \hat{v}_{k}$ will be designed in such a way that the nominal control $u_{k}^{n}=-\bar{L} \hat{\bar{x}}_{k}$ of the jump-free system (1) (obtained by a



FigURE 20: GLR test applied to adaptive algorithm with presence of two sequential faults.

LQG approach on an infinite horizon) is reconfigured online after each detection and isolation of one impulsive jump by the additive term $G \hat{v}_{k}$. In order to design $G$ in relation with the available nominal control law, we assume that the implementation of the passive GLR test is based on the twostage Kalman filter, the only optimal filter which gives the state prediction of the jump-free system $\hat{\bar{x}}_{k}$. So, let

$$
u_{k}=u_{k}^{n}-G v_{k}
$$

be the control law that we wish to design for a physical rejection of jumps $v_{k}$. Under the state transformation

$$
\left[\begin{array}{c}
\bar{x}_{k} \\
\nu_{k}
\end{array}\right]=\left[\begin{array}{ll}
I & T \\
0 & I
\end{array}\right]\left[\begin{array}{l}
x_{k} \\
\nu_{k}
\end{array}\right],
$$

the system (49) with (51) can be expressed as

$$
\begin{gathered}
{\left[\begin{array}{c}
\bar{x}_{k+1} \\
\nu_{k+1}
\end{array}\right]=\left[\begin{array}{cc}
A & (I-A) T+F \\
0 & I
\end{array}\right]\left[\begin{array}{l}
\bar{x}_{k} \\
\nu_{k}
\end{array}\right]+\left[\begin{array}{l}
B \\
0
\end{array}\right]\left(u_{k}^{n}-G v_{k}\right),} \\
y_{k}=\left[\begin{array}{ll}
C & -C T
\end{array}\right]\left[\begin{array}{l}
\bar{x}_{k} \\
\nu_{k}
\end{array}\right],
\end{gathered}
$$

and the physical rejection of jumps will be obtained if and only if $T$ and $G$ satisfy the algebraic equations

$$
\begin{gathered}
(I-A) T+F=-B G, \\
C T=0 .
\end{gathered}
$$

Under the existence condition of a solution of (55), (56) given by

$$
\operatorname{rang}\left[\begin{array}{ccc}
A-I & B & -F \\
C & 0 & 0
\end{array}\right]=\operatorname{rang}\left[\begin{array}{cc}
I-A & B \\
C & 0
\end{array}\right]
$$




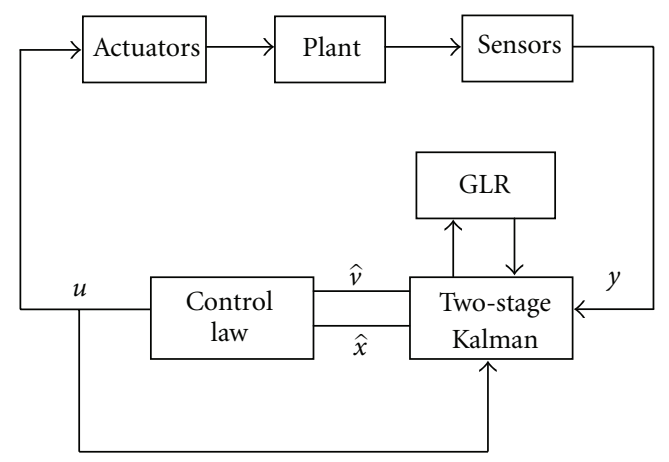

FIgUre 21: The reconfigurable FTCS scheme based on the passive GLR test.

the gain $G$ of the control law (51), solution of (55) gives

$$
G_{1}=\left[C(I-A)^{-1} B\right]^{-1} C(I-A)^{-1} F
$$

and $T=(I-A)^{-1}(B G-F)$ is not used. Under (55), (53) gives

$$
\begin{gathered}
{\left[\begin{array}{l}
\bar{x}_{k+1} \\
v_{k+1}
\end{array}\right]=\left[\begin{array}{ll}
A & 0 \\
0 & I
\end{array}\right]\left[\begin{array}{l}
\bar{x}_{k} \\
v_{k}
\end{array}\right]+\left[\begin{array}{l}
B \\
0
\end{array}\right] u_{k}^{n}++\left[\begin{array}{l}
I \\
0
\end{array}\right] w_{k},} \\
y_{k}=\left[\begin{array}{ll}
C & 0
\end{array}\right]\left[\begin{array}{l}
\bar{x}_{k} \\
v_{k}
\end{array}\right]+v_{k},
\end{gathered}
$$

where the jump-free state $\bar{x}_{k}$ of the system is decoupled to the jump's states $v_{k}$ and where $u_{k}^{n}=-\bar{L}_{\bar{x}}$ can then be considered as the nominal control law assumed to be designed on the jump-free system (1). From the well-known separation principle, the reconfigurable control law, which reject the $N$ jump's uncontrollable modes, is then derived

$$
u_{k}=-\bar{L} \hat{\bar{x}}_{k}-G \hat{v}_{k}
$$

where $\hat{\bar{x}}_{k}$ is the state prediction of the jump-free system and $\hat{\nu}_{k}$ the bias magnitude prediction given by the two-stage Kalman filter of Friedland [22] which optimally implement (34) (see Figure 21), (see the Appendix).

The last scheme resumes the control strategy to compensated automatically fault effects. The reference model used for the design of the control law GLR test coincides with the reference model used by the GLR Test. After each detection and isolation of one jump, the reference model is updated with the new state of jump and the three parts of the FTCS, that is, the GLR detector, the Kalman filter, and the control law.

\section{Conclusion}

Derived from the works of Willsky and Jones [1], this paper has presented the passive GLR test for sequential jumps detection in stochastic discrete-time linear systems. Based on a new adaptive augmented state filtering designed on the least favorable faulty model of the system, the passive GLR test gives a good results on detection and isolation of multiple faults, and it can be integrated very easily in a fault tolerant control System.

\section{Appendix}

The predictive form of the Freidland's two-stage Kalman filter which optimally implement the augmented state Kalman filter (34) is given by

$$
\begin{gathered}
\hat{x}_{k+1}=\hat{\bar{x}}_{k+1}+\zeta_{k+1} \hat{v}_{k+1}, \\
P_{k+1}=\bar{P}_{k+1}+\zeta_{k+1} P_{k+1}^{v} \zeta_{k+1}^{T},
\end{gathered}
$$

where $\hat{\bar{x}}_{k+1}$ and $\bar{P}_{k+1}$ are given by the bias-free filter (1) and where $\hat{\nu}_{k}$ and $P_{k}^{v}$ are given by the bias filter

$$
\begin{gathered}
\hat{v}_{k+1}=\hat{v}_{k}+K_{k}^{v}\left(\bar{\gamma}_{k}-\varrho_{k} \hat{\nu}_{k}\right), \\
K_{k}^{v}=P_{k}^{v} \varrho_{k}^{T}\left(\bar{H}_{k}+\varrho_{k} P_{k}^{v} \varrho_{k}^{T}\right)^{-1}, \\
P_{k+1}^{v}=P_{k}^{v}-P_{k}^{v} \varrho_{k}^{T}\left(\bar{H}_{k}+\varrho_{k} P_{k}^{v} \varrho_{k}^{T}\right)^{-1} P_{k}^{v} \varrho_{k} .
\end{gathered}
$$

From the coupling equation

$$
\begin{gathered}
\zeta_{k+1}=\left(A-\bar{K}_{k} C\right) \zeta_{k}+F, \\
\varrho_{k}=\zeta_{k},
\end{gathered}
$$

the initial conditions of the two-stage Kalman filter must be fixed as follows:

$$
\begin{gathered}
{\left[\begin{array}{l}
\hat{\bar{x}}_{0} \\
\hat{\nu}_{0}
\end{array}\right]=\left[\begin{array}{cc}
I & -\zeta_{0} \\
0 & 1
\end{array}\right]\left[\begin{array}{l}
\hat{\bar{x}}_{0} \\
\hat{\nu}_{0}
\end{array}\right],} \\
{\left[\begin{array}{cc}
\bar{P}_{0} & 0 \\
0 & P_{0}^{v}
\end{array}\right]=\left[\begin{array}{cc}
P_{0}^{x} & P_{0}^{x \nu} \\
P_{0}^{\nu x} & P_{0}^{v}
\end{array}\right]\left[\begin{array}{cc}
I & -\zeta_{0} \\
0 & 1
\end{array}\right]^{T}}
\end{gathered}
$$

with $\zeta_{0}=P_{0}^{x \nu}\left(P_{0}^{\nu}\right)^{-1}$.

\section{References}

[1] A. S. Willsky and H. L. Jones, "A generalized likelihood ratio approach to the detection and estimation of jumps in linear systems," IEEE Transactions on Automatic Control, vol. 21, no. 1, pp. 108-112, 1976.

[2] A. S. Willsky, "A survey of design methods for failure detection in dynamic systems," Automatica, vol. 12, no. 6, pp. 601-611, 1976.

[3] J. C. Deckert, M. N. Desai, J. J. Deyst, and A. S. Willsky, "F-8 DFBW sensor failure identification using analytical redundancy," IEEE Transactions on Automatic Control, vol. 22, no. 5, pp. 795-804, 1977.

[4] D. E. Gustafson, A. S. Willsky, and J.-Y. Wang, "ECG/VCG rhythm diagnosis using statistical signal analysis. I. Identification of persistent rhythms," IEEE Transactions on Biomedical Engineering, vol. 25, no. 4, pp. 344-353, 1978.

[5] M. Basseville and A. Benveniste, "Design and comparative study of some sequential jump detection algorithms for digital signal," IEEE Transactions on Acoustics, Speech, and Signal Processing, vol. 31, no. 3, pp. 521-535, 1983. 
[6] A. S. Willsky, E. Y. Chow, S. B. Gershwin, C. S. Greene, A. L. Kurkjian, and P. K. Houpt, "Dynamic model-based techniques for the detection of incidents on freeways," IEEE Transactions on Automatic Control, vol. 25, no. 3, pp. 347-360, 1980.

[7] J.-Y. Keller, "Fault isolation filter design for linear stochastic systems," Automatica, vol. 35, no. 10, pp. 1701-1706, 1999.

[8] R. V. Beard, Failure accommodation in linear systems through self-reorganisation, Ph.D. thesis, MIT Man Vehicle Laboratory, Cambridge, Mass, USA, 1971.

[9] R. J. Patton, "Fault-tolerant control: the 1997 situation," in Proceedings of the IFAC Symposium on Fault Detection, Supervision and Safety for Technical Processes (SAFEPROCESS '97), pp. 1033-1055, Hull, UK, 1997.

[10] J. Chen and R. J. Patton, Robust Model-Based Fault Diagnosis for Dynamic Systems, Kluwer Academic Publishers, Norwell, Mass, USA, 1999.

[11] D. P. Looze, J. L. Weiss, J. S. Eterno, and N. M. Barrett, "An automatic redesign approach for control methods," IEEE Control Systems Magazine, vol. 5, no. 2, pp. 16-22, 1985.

[12] N. E. Wu, Y. Zhang, and K. Zhou, "Control effectiveness estimation using an adaptive Kalman estimator," in Proceedings of the IEEE International Symposium on Intelligent Control (ISIC '98), pp. 181-186, September 1998.

[13] J. Jiang, "Design of reconfigurable control systems using eigenstructure assignments," International Journal of Control, vol. 59, no. 2, pp. 395-410, 1994.

[14] P. S. Maybeck and R. D. Stevens, "Reconfigurable flight control via multiple model adaptive control methods," IEEE Transactions on Aerospace and Electronic Systems, vol. 27, no. 3, pp. 470-480, 1991.

[15] M. Bodson and J. E. Groszkiewicz, "Multivariable adaptive algorithms for reconfigurable flight control," IEEE Transactions on Control Systems Technology, vol. 5, no. 2, pp. 217-229, 1997.

[16] A. K. Caglayan, S. M. Allen, and K. Wehmuller, "Evaluation of second generation reconfiguration strategy for aircraft flight control systems subject to actuator failure/surface damage," in Proceeding of the IEEE National Aerospace and Electronics Conference (NAECON '88), pp. 520-529, New York, NY, USA.

[17] C. Y. Huang and R. F. Stengel, "Restructurable control using proportional-integral implicit model following," Journal of Guidance, Control, and Dynamics, vol. 13, no. 2, pp. 303-309, 1990.

[18] M. Mahmoud, "Stabilizing controllers for a class of discrete time fault tolerant control systems," ICIC Express Letters, vol. 2, no. 3, pp. 213-218, 2008.

[19] M. Mahmoud, "Sufficient conditions for the stabilization of feedback delayed discrete time fault tolerant control systems," International Journal of Innovative Computing, Information and Control, vol. 5, no. 5, pp. 1137-1145, 2009.

[20] R. Youssef and P. Hui, "Piecewise sliding mode decoupling fault tolerant control system," ICIC Express Letters, vol. 4, no. 4, pp. 1215-1222, 2010.

[21] S. Tong, T. Wang, and W. Zhang, "Fault tolerant control for uncertain fuzzy systems with actuator failures," International Journal of Innovative Computing, Information and Control, vol. 4, no. 10, pp. 2461-2474, 2008.

[22] B. Friedland, "Treatment of bias in recursive filtering," IEEE Transactions on Automatic Control, vol. 14, pp. 359-367, 1969.

[23] S. Tanaka, Diagnosability of Systems and Optimal Sensor Location, International Series in Systems and Control Engineering, Prentice Hall, London, UK, 1990.
[24] A. K. Caglayan, "Necessary and sufficient conditions for detectability of jumps in linear systems," IEEE Transactions on Automatic Control, vol. 25, no. 4, pp. 833-834, 1980.

[25] B. D. O. Anderson and J. B. Moore, Optimal Control: Linear Quadratic Methods, Prentice Hall, Englewood Cliffs, NJ, USA, 1990.

[26] A. S. Willsky, "Detection of abrupt changes in dynamic systems," in Detection of Abrupt Changes in Signals and Dynamical Systems, Lecture Notes in Control and Information Sciences, pp. 27-49, Springer, New York, NY, USA, 1986.

[27] M. Basseville and I. V. Nikiforov, Detection of Abrupt Changes Theory and Application, Prentice Hall, Thomas Kailath, Englewood Cliffs, NJ, USA, 1994. 

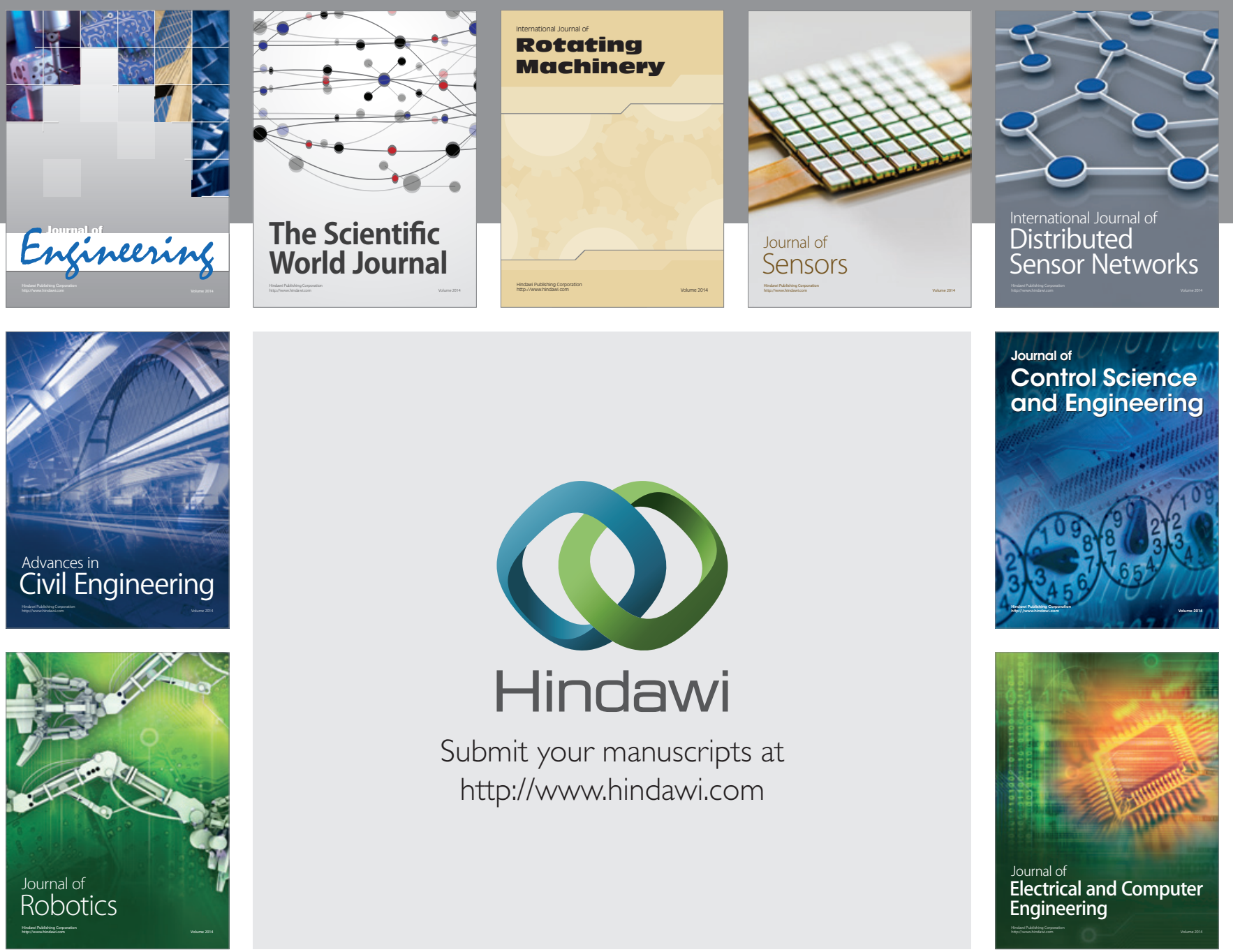

Submit your manuscripts at

http://www.hindawi.com
